\title{
Toward a Better Understanding of Helicopter Stability Derivatives
}

\section{Raymond S. Hansen}

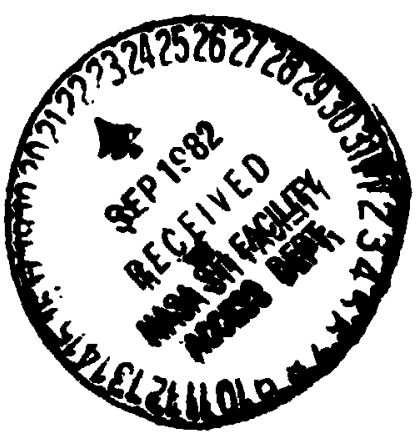




\section{Toward a Better Understanding of Helicopter Stability Derivatives}

Raymond S. Hansen, Aeromechanics Laboratory
AVRADCOM Research and Technology Laboratories
Ames Research Center, Motfett Field, California 


\title{
EIGHTH EUROPBAN ROTORCRAFT FORUY
}

\section{Lichten Award Paper}

TOWARD A BETTER UNDERSTANDING OF HELICOPTER STABILITY DERIVATIVES

\author{
Raymond S. Hansen
}

U.S. Army Aeromechanics Laboratory NASA Ames Research Center

Mof fett Field, California 94035 U.S.A.

August 31 through September 3, 1982 Alx-En-Provence, FRANCE 
TOWARD A BETTER UNDERSTANDING OF HELICOPTER STABILITY DERIVATIVES

\author{
Raymond S. Hansen \\ U.S. Army Aeromechanics Laboratory \\ NASA Ames Research Center \\ Moffett Field, Callfornia 94035 U.S.A.
}

\begin{abstract}
An amended six-degree-of-freedom helicopter stability and control derivative model was developed in which body acceleration and control-rate derivitives were included in the Taylor serles expansion. These additional derivitives were derived from consideration of the effects of the higher-order rotor-f lapping dynamics, which are known to be inadequately represented in the conventional six-degree-of-freedom, quasi-static stability-derivative model. The amended model was found to be a substantial improvement over the conventional model, effectively doubling the usable bandwidth and providing a more accurate representation of the short-period and cross-axis characteristics. Further investigations assessed the applicability of the two stabilityderivative model struclures for flight-test parameter identification. Parameters were identified using simulation data generated from a higher-order baseline model having sixth-order rotor tip-path-plane dynamics. Three lower-order models were identified: one using the con'entional stability-derivative model structure, a second using the amended six-degree-of-freedom model structure, and a third model having eight degrees of freedom that included a simplified rotor tip-path-plane tilt representation.
\end{abstract}

\title{
LIST OF SYMBOLS
}

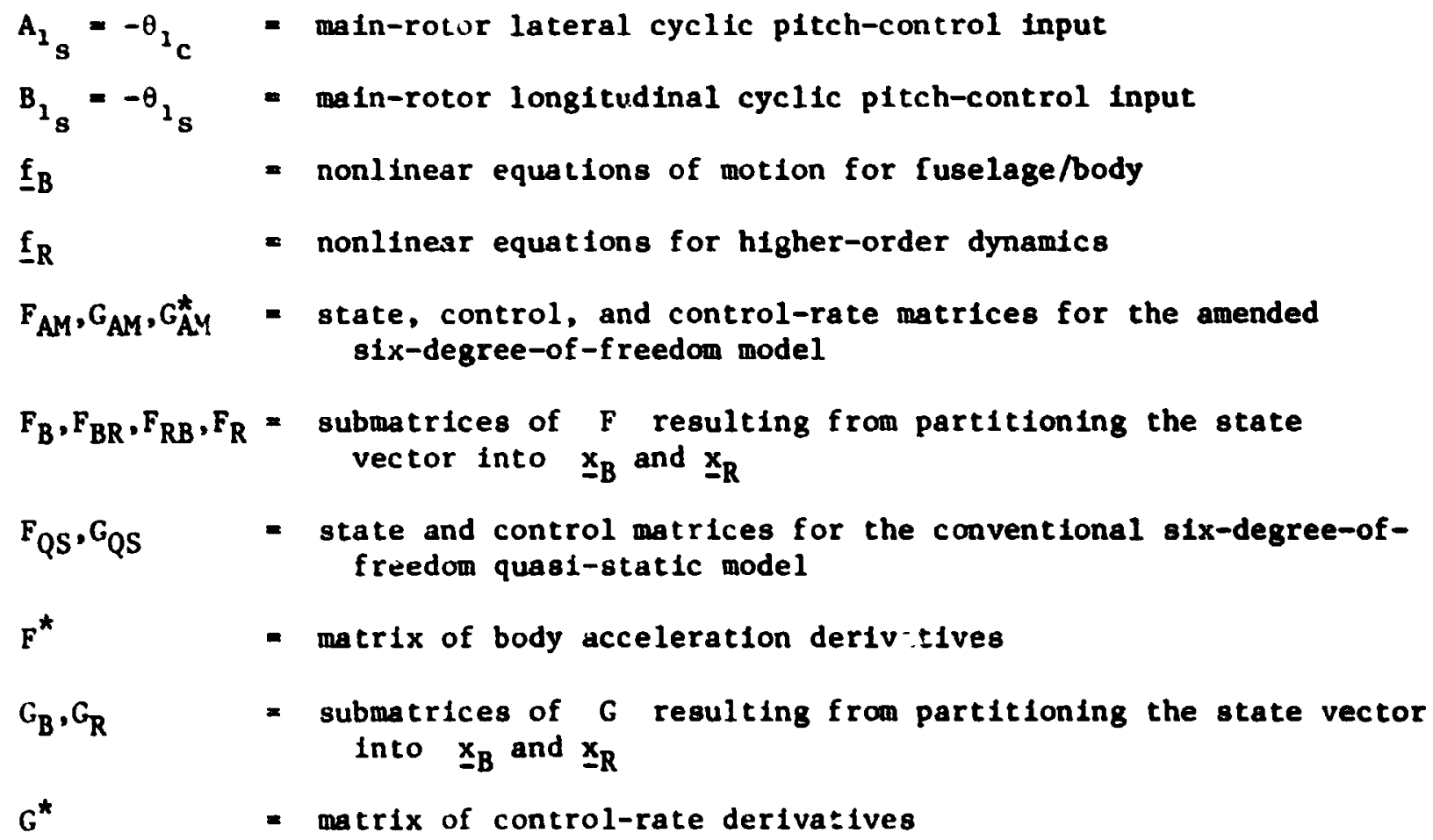


$I_{\mathbf{y}}$

L( )

M( )

N( )

$p, q, r$

$\mathbf{s}$

t

$u, v, w$

$\underline{\mathbf{u}}$

$\underline{x}_{\mathbf{B}}$

$\underline{x}_{R}$

$\underline{x}_{R_{r}} \cdot \underline{x}_{R_{b}}$

$\left.x_{(}\right)$

Y ( )

Z( )

B

$\delta$

$\Delta()$

$\theta$

$\theta_{\mathrm{b}}$

${ }^{\theta} \mathrm{TR}$

$\phi$

$\psi$

()$^{-1}$

()$^{\mathrm{T}}$

$\left({ }^{\circ}\right)$
- identity matrix

- pitch-axis moment of inertia

- rolling-moment derivat: se

- pitching-moment derivative

- yawing-moment derivative

- roll, pitch, and yaw rates

= Laplace operator

- time

= longltudinal, lateral, and vertical velocities

- control vector

$=$ fuselage/body state vector $=[\Delta u \Delta v \Delta w \Delta p \Delta q \Delta r \Delta \phi \Delta \theta]^{T}$

= state vector for all higher-order dynamics; also can reprusent any one of several simplified rotor models (see Table 1)

- components of decomposed rotor response

- longitudinal force derivative

- lateral force derivative

- vertical force derivative

- blade-flapping angle, for ith blade:

$\beta_{1}=\beta_{0}+\beta_{1} \cos \psi_{1}+\beta_{1}$ sin $\psi_{1}$ (tip-path-plane assumption)

- elevator deflection

- Indicates perturbation of quantity in parenthesis

- body pitch attitude

- blade pitch angle, for ith blade:

$\theta_{b_{1}}=\theta_{0}+\theta_{1} \cos \psi_{1}+\theta_{1}$ sin $\psi_{1}$

- tail-rotor collective pitch input

- body roll attitude

- main-rotor blade azimuth position, $(\psi=0$ is af $t)$

- matrix Inverse

- matrix transpose

- time derivative 


\section{Introduction}

Stability and control derivatives are used for evaluating helicopter flight dynamics about a given operating point or trim condition. They are a source of information about amall-perturbation stability and reeponse to control inputs, and are necessary for a wide varlety of applications, lacluding handling-qualities analysis, flight-control aystems design, and real-time simulation.

Stability and control derivatives are attributed to Bryan (Ref. (1]) who, In 1911, developed them to investigate the stability of airplanes. Uaing his method, the change in an aerodynamic force or moment assoclated with each of the primary six degrees of freedom (6 DOF) of an alrcraft is expressed as a Taylor series consisting of perturbation terms in each of the six primary eotion states and the controls. The series is truncated such that only the linear terms are retained in the stability-derivative expansion. In the years ance, airplane flight dynamiclsts recognized that additional unmodeled degrees of freedom above the basic six contrlbute to aircraft flight dynamic behavior. of significance are air-mass dynamics, which cause a lag in downash at the horizontal tall, and control-surface dynamics, which are necessary for evaluating alrcraft stick-free stability. In order to approximate these ummodeled degrees of freedom, flight dynamicists selectively introduced body acceleration and control-rate derivatives, such as $M_{j}, z_{\dot{W}}$, and $M_{j}$ into the stability-derivative expansion.

Helicupter flight dynamicists have traditionally used the same stabilityderivative expansion formulation as that used for airplane analysis (except that asymetric cross-coupling derivatives have been retained), and have justifled their action based on the assumption of the quasi-static perturbation derivative. The perturbation derivative for the change in pitching moment resulting from a change in vertical velocity, for example, is defined as:

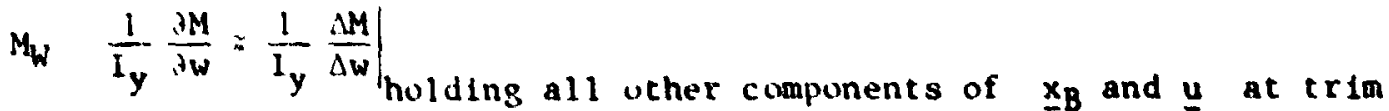

$$
\begin{aligned}
& \text { value; allowing } \underline{x}_{R} \text { to reach new equilib̈rium }
\end{aligned}
$$

where the change in pitching moment $(M M)$ owing to an instantaneous change in vertical velocity $(\Delta w)$ is calculated by suppressing the integration of the body states $\left(x_{B}\right)$, holding the controls (u) at the trim value, and allowing the higher-order rotor degrees of freedom $\left(\underline{x}_{R}\right)$ to reach their new equilibrium. The quasi-static stability derivative is the steady-state $A M$ divided by both the constant-perturbation $\Delta w$ and the pitch-axis inertia $I_{y}$. (To be strictly correct, the stability derivative is the limit of this quantity as $\Delta w \rightarrow 0$; however, practical considerations generally require an assessment of linearity by varying the size of $j_{w}$.)

Figure 1 demonstrates the $A M$ "response" resultirig from a constantperturbation $\Lambda w$ for several systems. For an ideal six-degree-of-freedom system, the IM response is instantaneous and time-invariant, indicating that the system can be perfectly represented using the stability-derivative formulation of Bryan. For an airplane, the response is very similar. There may be some inithal translents because of air-mass dynamics or, perhaps, structural wing bending: however, for most praitlcal purposes, alrplane flight dynamics can be well modeled by the constant-cocfficient stability derivatives. Inclusion of body accoleration and control-rate derivatives to approximate the inftial translents in $I M$ is generally an altempt to fine-tune an already good model. 
For a helicopter, Fig. 1 shows a significant initial transient lasting about $0.25 \mathrm{sec}$ as the rotor flaps to its new equilibrium position. The $\Delta M$ response is made up of two distinct contributions - first, an instantaneous contribution owing to body and tail aerodynamics, as well as rotor shears transmitted via the blade-hinge of $f$ set, and a second transient contribution resulting from all higher-order degrees of freedom (above the basic six) achieving a new equilibrium. The quasi-static stability derivative, as advanced by Hohenemser in 1939 (Ref. [2]), assumes that the rotor instantaneously reaches its new equilibrium, as is indicated by the dotted line in Fig. 1. The figure shows that from a strictly 6-DOF point of view, the perturbation derivative is actually time-varying. The conventional six-degree-of-freedom stahility-derivative model, which has the time-invariant quasi-static stability and control derivatives as its elements, is a poor approximation, because the dynamics of the rotor response are not well separated from those of the basic airframe dynamics.

Use of the conventional quasi-static stability-derivative model is adequate for many applications associated with low-frequency (such as phugoid) and steady-state filght-dynamic behavior; however, it is of ten $n_{1}$ : representative of the higher frequency short-period dynamics, owing to the strong influence of the unmodeled rotor modes. In the literature, Ellis (Ref. [3]) discusses the shortcomings of using the 6-DOF quasi-static model for design analyses of angular rate and attitude feedback systems. He concluded that use of this conventional model would result in inaccurate estimates of the stability boundaries for high-gain feedback systems, and would lead to an overly optimistic appraisal of true system capabilities. I.. an extensive control system design effort for the AH-56A (Ref. [4]), it is stated that the use of the 6-DOF quasistatic derivatives is found to give a deceptive impression of greater aircraft stability, because the regressing flapping mode is neglected. In Ref. [5], two optimal controllers are designed, based on a 6-DOF quasi-static model and on a model that included the rotor tip-path-plane tilt dynamics. It was concluded that for very tight control, rotor dynamics should be included in designing such controllers.

Attempts to extract stability and control derivatives from flight data have raised further questions about the validity of quasi-static derivatives. Molusis (Ref. [6]) has indicated that identified derivatives can take on considerably different values from those of the analytic perturbation derivatives, owing to the fact that in flight, the rotor is continuously being excited by pilot control inputs and turbulence, and is not operating in a quasi-static or steady-state fashion. Reference [7] also indicates that for a Bo-105 hel1copter, there are significant discrepancies between filght-identified values and manufacturer-supplied analytic and wind-tunnel startup values. Increasing the a priori weighting made the identified derivatives more consistent with the startup values, but only at the expense of degraded curve fits with the flight data. In Ref. [8], Gould and Hindson incorporated selected body acceleration and control-rate derivetives in an identification of the lateras directional stability characteristics of a teetering-rotor helicopter. In their study, these derivatives were introduced by assuming that the lags 1 . the main-rotor tip-path-plane response and the side-wash at the tail rotor can each be approximated by first-order time-constants. No general theory for body-acceleratina and control-rate derivatives exists in the 11terature, and the implications of including these terms in the stability-derivative expanston are not clearly understood.

In spite of all its problems, use of the conventional stabilityderivative formulation persists in the helicopter community. Ferhaps this 18 
because linear rotor + body models ace not readily derived by analytic means and must be extracted from the more comprehensive nonlinear models. More likely, it persists because there is a certain loss of physical interpretation that goes with the higher-order rotor + body models. Except in those cases in which it is clearly necessary to go with a higher-order model, it is preferred to stay in the 6-DOF domain. Because of this, it is necessary to develop a better understanding of the limitations of the conventional model, and to investigate potential improvements thet can extend its applicability.

\section{Helicopter Linear Modeling}

\section{General Formulation}

The nonlinear differential equations of motion for the dynamics of any flight vehicle can be written in a partitioned state-vector notation as follows:

$$
\begin{aligned}
& \underline{\dot{x}}_{B}=\underline{f}_{B}\left(\underline{x}_{B}, \underline{x}_{R}, \underline{u}, t\right) \\
& \underline{\dot{x}}_{R}=\underline{f}_{R}\left(\underline{x}_{B}, \underline{x}_{R}, \underline{u}, t\right)
\end{aligned}
$$

where the first vector differential equation represents the dynamics of the basic six degrees of fuselage/body motion, and the second vector equation represents all higher-order dynamics that may be of significance. For a helicopter, the second equation would, in its most general form, include rotorblade dynamics (e.g., flapping, lead-lag, and torsion), inflow/air-mass dynamics, control-system dynamics, and bending modes).

Linearization of the nonlinear equations results in equations that are periodic with rotor azimuth. A constant-coefficient formulation is obtained by averaging each periodic ierm over one rotor revolution. Further discussion of the linearization of nonlinear equations for helicopter flight dynamics can be found in Refs. [9] and [10].

\section{Rotor + Body Models}

Linearization and averaging of the nonlinear equations (1) and (2) will resulc in the following linear matrix equations of motion:

$$
\begin{aligned}
& \underline{\dot{x}}_{B}=F_{B} \underline{x}_{B}+F_{B R \underline{x}_{R}}+G_{B} \underline{u} \\
& \underline{\dot{x}}_{R}=F_{R B} \underline{x}_{B}+F_{R \underline{x}_{R}}+G_{R} \underline{u}
\end{aligned}
$$

where

$$
\begin{aligned}
\underline{x}_{B} & =\left[\Delta u \Delta v \Delta w \Delta p \Delta_{q} \Delta r \Delta \phi \Delta \theta\right]^{T} \\
\underline{u} & =\left[\Delta B_{1_{s}} \Delta A_{1_{s}} \Delta \theta_{T R} \Delta \theta_{0}\right]^{T} \\
\underline{x}_{R} & =\text { state vector for higher-oro - dynamics }
\end{aligned}
$$

Equations (3) and (4) are partitioned in order to specifically break out the individual effects of the rotor and the body. The $F_{R}$ and $G_{R}$ matrices alone represent the tynamics of the isolated rotor (in a wind tunnel, for instance). 
The $F_{B}$ and $G_{B}$ matrices represent the body-only aerodynamic effects that occur in the absence of rotor-flapping dyamics. (Direct hub forces and moments acting at the rotor head are included in these matrices.) The $\mathrm{F}_{\mathrm{BR}}$ and $\mathrm{P}_{\mathrm{BB}}$ matrices account for the rotor-to-body and the body-to-rotor coupling, respectively.

For many flight-dynamic applications, only the rotor-flepping degrees of freedom need to be considered in the $x_{R}$ vector. Table 1 shows several typical rotor + body models. The nine-degree-of-freedom (9-DOF) model incorporates the rotor as a three-degree-of-f reedom tip-path-plane, with each degree of freedom represented by a second-order differential equation. Th1s results in a sixth-order rotor-flapping system of equations which have three periodic normal modes of motion: the advaring flapping mode, the coning mode, and the regressing flapping mode.

The advancing (progressing) flapping mode, its frequency being roughly twice the rotor rotational frequency, is not of significance for helicopter flight dynamics (Ref. [11]) and should be eliminated; only the coning and regressing modes should be retained. The coning model, as it is called, still has 9 DUF, but the longitudinal and lateral tip-path-plane tilt equations are now each of first order and couple to give the regressing flapping mode. The coning mode is generally only significant for those helicopters that have low rotor rotational rates, that is, for high gross weight or slowed rotor vehicles. If the coning mode 18 also el iminated, one has the 8-DOF tip-pal'i-plane tilt model that retains only the regressing mode in addition tc the fust:lage/body modes.

\section{Stability Derivative Models}

Mathematical manipulation of equation (4) will yield a solution in terms of $\underline{x}_{B}, \underline{u}$, and higher-order derivatives. Taking the Laplace transform of equation ${ }^{-}(4)$ :

$$
s \underline{X}_{R}=F_{R B} \underline{X}_{B}+F_{R} \underline{X}_{R}+G_{R} \underline{U}
$$

Solving for $\underline{X}_{R}(s)$ gives,

$$
\underline{X}_{R}=\left(s I-F_{R}\right)^{-1}\left(F_{R B} \underline{X}_{B}+G_{R} U\right)
$$

The matrix inverse term $\left(s I-F_{R}\right)^{-1}$ may be expressed as an infinite series,

$$
\left(s I-F_{R}\right)^{-1}=-F_{R}^{-1}\left(I+F_{R}^{-1} s+F_{R}^{-2} s^{2}+F_{R}^{-3} s^{3}+\ldots \cdot\right)
$$

This series can be shown to converge absolutely in that region of the s-plane inside a circle of convergence centered at the origin with a radius equal to the magnitude of the smallest eigenvalue of $F_{R}$. That eigenvalue generally corresponds to the rotor regressing mode, and consequently the series is convergent for the range of frequencies of interest to the flight dynamicist.

Substituting equation (7) back into equation (6), and taking the inverse Laplace transform will yield the following solution to equation (4): 


$$
\underline{x}_{R}=-F_{R}^{-1} F_{R B} \underline{x}_{B}-F_{R}^{-1} G_{R} \underline{u}-F_{R}^{-2} F_{R B} \underline{x}_{B}-F_{R}^{-2} G_{R} \dot{u}-F_{R}^{-3} F_{R B} \ddot{x}_{B}-F_{R}^{-3} G_{R} \ddot{u} \ldots
$$

Equation (8) includes terms for the body states, the controls, and all their higher-order derivatives. It can be easily veriffed by substitution that it is a solution to equation (4).

Conventional Quasi-Static Model. The first two terms of the series shown in equation (8) are identical to the solution from the residualization method of model order reduction, where $\underline{\dot{x}}_{\mathbb{R}}$ is set equal to zero, and the rotor state vector $\underline{x}_{R}$ can be expressed as a function of $\underline{x}_{B}$ and $\underline{u}$ alone. This is equivalent to the quasi-static assumption discussed previousīy, where the rotor is assumed to reach its new equilibrium instantaneously, and can therefore be expressed in terms of the immediate body states and control positions:

$$
\underline{x}_{R}=-F_{R}^{-1} F_{R B} \underline{x}_{B}-F_{R}^{-1} G_{R} \underline{u}
$$

Substituting this approximate solution back into equation (3) results in the standard expression for the quasi-static model:

$$
\dot{\dot{x}}_{B}=\underbrace{\left(F_{B}-F_{B R} F_{R}^{-1} F_{R B}\right) \underline{x}_{B}}_{F_{Q S}}+\underbrace{\left(G_{B}-F_{B R} F_{R}^{-1} G_{R}\right) \underline{u}}_{G_{Q S}}
$$

The quasi-static state matrix $F_{O S}$ and control matrix $G_{O S}$ are defined as shown in terms of the fundamental rotor + body submatrices. The elements of the matrices $F_{Q S}$ and $G_{Q S}$ are identical to the conventiunal quasi-static perturiution derivatives, except that the $L, M$, and $N$ derivatives now reflect the effects of vehicle cross-products of inertia, and the usual linearized inertial terms rave been included.

Amended 6-DOF Model. The conventional model can be improved by including the third and fourth terms of equation (8) in the solution:

$$
\underline{x}_{R}=-F_{R}^{-1} F_{R B} \underline{x}_{B}-F_{R}^{-1} G_{R} \underline{u}-F_{R}^{-2} F_{R B} \underline{\underline{x}}_{B}-F_{R}^{-2} G_{R} \underline{u}
$$

The use of those two additional terms is equivalent to including bodyacceleration and control-rate derivatives in the solution for the higher-order dynamics. Substituting this solution for the rotor into equation (3) yields the solution

$$
\underline{\dot{x}}_{B}=F_{Q S} \underline{x}_{B}+G_{Q S} \underline{u}+\underbrace{\left(-F_{B R} F_{R}^{-2} F_{R B}\right)}_{F^{*}} \dot{\underline{x}}_{B}+\underbrace{\left(-F_{B R} F^{-2} G_{R}\right) \underline{\dot{u}}}_{G^{*}}
$$

where the body-acceleration matrix $F^{*}$ and control-rate matrix $G^{*}$ are defined as shown. It should be noted that in the general case in which $\underline{x}_{R}$ includes the effects of the rotor dynamics (i.e., blade flapping, lead-lag, and torsion) a.d the air-mass dynamics (1.e., inflow, lag in downwash, etc.), contributions are introduced into the $\mathrm{F}^{*}$ and $\mathrm{C}^{*}$ matrices from $\mathrm{all}$ of these sources. If a rotor + body model is used as a basis for generating the amended model matrices, on $1 y$ the "pseudo" unsteady derfvatives coming about because of rotor-flapping dynamics alone will be reflected in the $F^{*}$ and $G^{*}$ matrices. 
Equation (12) can be rewritten into an alternative form by grouping the body-acceleration terms to give

$$
\dot{\underline{x}}_{B}=\underbrace{\left(I-F^{*}\right)^{-1} F_{Q S} \underline{x}_{B}}_{F_{A M}}+\underbrace{\left(I-F^{*}\right)^{-1} G_{Q S} \underline{u}}_{G_{A M}}+\underbrace{\left(I-F^{*}\right)^{-1} G^{*} \dot{\underline{u}}}_{G_{A M}^{*}}
$$

where the matrices $F_{A M}, G_{A M}$, and $G_{A M}^{*}$ are defined as shown.

Rotor-Response Decomposition Models

Rotor-response decomposition models are an intermediate formulation falling between the rotor + body models and the stability-derivative models. The Idea is to decompose the response of the higher-order states into a response that is self-induced and a response that is forced by the body motions. Decomposing $\underline{x}_{R}$ as follows,

$$
\underline{x}_{R}=\underline{x}_{R_{r}}+\underline{x}_{R_{b}}
$$

where $\underline{x}_{R_{r}}$ is the response owing to - stor motions and $\underline{x}_{R_{b}}$ is the response owing to body motions. The differential equation (4) then splits to give

$$
\begin{aligned}
& \underline{\dot{x}}_{R_{r}}=F_{R^{x_{R}}}+G_{r} \underline{u} \\
& \underline{\dot{x}}_{R_{b}}=F_{R^{x_{R}}}+F_{R B} \underline{x}_{B}
\end{aligned}
$$

A solution for $\underline{x}_{R_{b}}$ can be written using the same approach as for equation (8).

$$
\underline{x}_{R_{b}}=-F_{R}^{-1} F_{R B} \underline{x}_{B}-F_{R}^{-2} F_{R B} \underline{\underline{x}}_{B}-I T_{R}^{-3} F_{R B} \ddot{x}_{B}-\cdots
$$

If only the first term is retained, the assumption is that the body dynamics are slow compared with the rotor motions, and the rotor response to the iody motions only are assumed to be instantaneous. The rotor state vector $\underline{x}_{R}$ can the $t$ be written as

$$
\underline{x}_{R}=\underline{x}_{R_{r}}-F_{R}^{-1} F_{R B} \underline{x}_{B}
$$

Substituting this into equation (3) yields

$$
\begin{aligned}
& \underline{\dot{x}}_{B}=F_{Q S \underline{x}_{B}}+F_{B R \underline{x}_{R_{r}}}+G_{B \underline{u}} \\
& \underline{\dot{x}}_{R_{r}}=F_{R} \underline{x}_{R_{r}}+G_{R} \underline{u}
\end{aligned}
$$

where $F_{Q S}$ is the atate matrix derived for the conventional quasi-static model in equation (10). An approximation similar to this was used in Ref. [3], except that the $G_{B}$ u term was neglected. When this 18 the case, the combined rotor + body dynamics can be treated as the product of two transfer functions, that is, a rotor transfer function $Y_{Y_{r}}(s) / \underline{U}(s)$ multiplying a body transfer function $\underline{X}_{B}(s) / \underline{X}_{R_{r}}(s)$ (notation used ctually implies multiplication of transfer function : atrices). 
If the second term of equation (17) is included in the solution (as was done in the amended model formulation), a solution to $x_{R}$ including body acceleration terms can be written:

$$
\underline{x}_{R}=\underline{x}_{R_{r}}-F_{R}^{-1} F_{R B} \underline{x}_{B}-F_{R}^{-2} F_{R B} \underline{x}_{B}
$$

The differential equations then become

$$
\begin{aligned}
\dot{\underline{x}}_{B} & =F_{A M} \underline{X}_{B}+\left(I-F^{*}\right)^{-1} F_{B R} \underline{X}_{R_{r}}+\left(I-F^{*}\right)^{-1} G_{B \underline{U}} \\
\underline{\dot{x}}_{R_{r}} & =F_{R^{\prime} \underline{x}_{R_{r}}}+G_{R} \underline{u}
\end{aligned}
$$

where $F_{A M}$ is identical to that state matrix used in the amended model equation (13).

Equations (22) and (23) may provide a particularly good model structure for combined rotor + body parameter identification. The isolated rotor equation (23) might already be well known or could be obtained from rotor testing in a wind tunnel. The matrices in equation (22) could thell be identified from flight testing. (Note, however, that $\underline{x}_{R_{r}}$ is not directly measurable.)

\section{Evaluation of Linear Flight Dynamics Models}

Method of Approach

Since the principal purpose of this study is to assess tie effects of the rotor-flapping dynamics on the 6-DOF stability derivatives, a 9-DOF helicopter model was chosen as the baseline model - the "absolute" with which all lowerorder models are to be compared. The model was intentionally chosen to be linear, in order to avold any problems in differentiating between linear and nonlinear effects, and to have only the rotor-flapping dynamics included as the higher-order modes, in order to assess their effect alone. The additional degrees of freedom not included (air-mass dynamics, inplane motions, etc.) are assumed to be quasi-statically lumped into this baseline structure. The model used was a linear fourteenth-order (sixth-order rotor) model of the $\mathrm{CH}-53 \mathrm{~A}$ at a 100-knot, level-flight trim condition (published in Ref. [12]).

The general approach, consisting of two parts, 18 shown in Fig. 2. The first involves model-o::der reduction, where the baseline model is matheratically reduced into three models - the conventional 6-DOF quasi-static stabilityderivative model, the amended 6-DOF model, and the 8-DOF t1p-path-plane tilt model. The objective is to evaluate the stability-derivative models against the baseline model and to directly assess the effects of the rotor dynamics on these models. The 8-DOF model is included because it is the lowest-urder rotor + body model and represents the next higher model in complexity above the stabilityderivative models.

The second part of the approach is a system-identification rpproach in which three models are identiffed from baseline model simulation data, using the structures of each of the evaluation models. The purpose 18 to see how well each of the models can be identifled in these idealized conditions. It 18 expected that the identified models would be sumewhat different from the reduced models, since the identification is a curve-fitting process that will be attempting to fit the baseline data with a lower-order model. 
The system-identification results are expected to provide guldelines for the interpretation of stability derivatives extracted from flight data. The identified models can be corsidered to represent an upper bound on the quality of results that can be obtained from flight testing; that bound will probably be somewhat optimistic considering the measurement and testing difficulties associated with parameter-identification flight experimentation.

Mathematica11y Reduced Mode1s

Reduced-order models for the conventional 6-DOF model, the amended 6-DOF model, and the 8-DOF tip-path-plane model were generated by mathematically reducing the baseline model. The baseline model was partitioned into its $F_{B}$, $F_{B R}, F_{R B}, F_{R}, G_{B}$, and $G_{R}$ matrices, and the $t w^{r}$ icability-derivative models were computed from equations (10) and (13), respecilvely. The 8-DOF model was generated using standard residualization techniques, whereby the baseline model is partitioned such that the residual vector is $\left[\Delta \beta_{0} \Delta \dot{B}_{0} \Delta \dot{\beta}_{1_{c}} \Delta \dot{\beta}_{1_{g}}\right]^{T}$, and the timederivative of this residual vector is set equal to zero.

Co-sparison of Time-History Responses. Time-histories from simulations of each of the three mathematically reduced models and the baseline model are shown in Fig. 3. The control excitation is a 2.1-rad/sec sinusoidal pitch axis $\left(B_{1 s}\right)$ inpit. Only plots for the primary-axis response (pitch rate) and the cross-axis (roll-rate) responses are shown in Fig. 3, for these satisfactorily represent the extremes of the comparison.

In the primary axis, the responses from all three reduced-order models closely resemble the baseline-model response. If the primary-axis response is considered alone. It could te concluded cnat the conventional 6-Dor quasi-static model is adequate for predicting handling-qualities behavior. However, there are considerable differences in the cross-axis response of the four models. Whereas the 8-DOF model does duplicate the baseline response, the mathematically reduced conventional model (having the usual stability-derivative formulation) does not. This conventional as itel incorrectly predicts the initial transient response in the first second, - id does not correctly predict the phase response for the subsenuent motion. Or: th s other hand, the amended 6-DOF model (which includes the $L$ Jy-acceleration and control-rate derivatives) does provide a more reasonable prediction of the roll-rate response, correctly predicting the initial response direction and the correct phase relationship; however, it does have some problems in predicting the correct amplitude behavior.

Frequency-Response Comparison. Phase and amplitude discrepancies in Fig. 3 prompted a closer inspection of the frequency-response characteristics of the reduced-order models, in order tu determine the usable bandwidth of each. (Usable bandwidth can be defined as that bandwidth for which a given model will duplicate the true frequency-response cnaracteristics.) Normalized amplitude and phase plots for two transfer functions are shown in Fig. 4. The pitch-rate response to a pitch-axis input (primary-axis response) and the roll-rate response to pitch-axis input (cross-axis response) have been normalized by the corresponding baseline-model responses. The baseline-model response is then indicated by the unity axis on the normalized amplitude plot and the zero axis on the relative phase plot. A frequency range from 0.2 to $5.0 \mathrm{rad} / \mathrm{sec} 1 \mathrm{~s}$ shown, with the phugoid frequency typically being between 0.2 and $0.5 \mathrm{rad} / \mathrm{sec}$ ), the Dutch roll between 1.0 and $2.0 \mathrm{rad} / \mathrm{sec}$, and the short period between 2.0 and $5.0 \mathrm{rad} / \mathrm{sec}$. 
Al though the reduced-order models all duplicate the low-frequency response characteristics of the baseline model, major discrepancies show up in the higher-frequency cross-axis response. The conventional model shows a departure from the baseline response at about $0.3 \mathrm{rad} / \mathrm{sec}$, the amended model at $0.8 \mathrm{rad} / \mathrm{sec}$, and the $8-\mathrm{DOF}$ model at about $1.3 \mathrm{rad} / \mathrm{sec}$. The usable bandwidth of the amended model is roughly twice that of the conventional quasi-static perturbation derivative model.

Eigenvalue Comparison. Characteristic roots for the basic 6-LOF fuselage/body motion are compared in Fig. 5. As expected, all the models correctly predict the low-frequency modes, with some slight differences showing up in the Dutch roll mode, and the significant discrepancies occurring in the short-period dynamics. Both the 8-DOF and amended models give a good accounting of the baseline short-period eigenvalues; however, the conventional model, although it correctly predicts the natural frequency, gives an optimistic estimate of the damping. Note that inclusion of the body-acceleration derivatives in the amended model is suffictent to modify the state matrix of the conventional model (i.e., $\left.F_{A M}=\left(I-F^{*}\right)^{-1} F_{Q S}\right)$ and to yield improved short-period eigenvalues without changing the low-frequency characteristics. This might lead one to conclude that the body-acceleration derivatives alone are responsible for the significant increase in the usable bandwidth of the amended model; however, that is not the case. Additional analyses of a model that included the body-acceleration terms but not the control-rate derivatives showed frequencyresponse characteristics comparable to those of the conventional model, indicat. ing that the control-rate derivatives are a necessary component of the amended model.

\section{Identified Models}

System identification from analytic models is discussed in Ref. [10]. The procedure used in this study consists of two parts - parameter identification and evaluation of the tdentified models.

Identification from Simulation Data. The baseli:ie model was excited using two representative inputs for each of the four control axes, the first beiug a doublet with a period of $3 \mathrm{sec}$ and the second a 3-2-1-1 input (intervals in seconds) that is of ten used for parameter identification because of wide-band excitatior (see Ref. [13]). The doublet was chosen specifically to excite the short-period aircraft dynamics. The amplitude used for both input waveforms in a11 axes was $0.02 \mathrm{rad}$. The eight maneuvers were simulated for $10 \mathrm{sec}$ each (a total of $80 \mathrm{sec}$ ) and were then sequentially processed, using a least-squares algorithm. Three sets of parameters were identified, using each of the reducedorder model structures.

The least-squares algorithm is the standard solution to the equationerror formulation of the parameter-identification problem and will, in the absence of measurement noise, yield optimum unbiased parameter estimates. Since there is no noise associated with the simulation itself, except perhaps negligible computer round-oif and integration routine errors, the least-squares algorithm is best for this application. All unmodeled higher degrees of freedom are treated as process noise (the assumption when using the equation-error formulation).

A large amount of data ( $30 \mathrm{sec}$ in all) was used to assure convergence of the parameters. It should be noted that using different control input waveforms and inter-axis amplitudes (i.e., relative amplitudes between control axes! is 
expected to yield slightly different converged parameter values. The convergence properties generally will depend on control-input waveform, amplitude. and spectral content, as well as on basic considerations, such as number of maneuvers, maneuver length, and sample rate. Investigation of all these factors was beyond the scope of this effort and remains an area for continuing research.

Evaluation of Identified Models. The crucial test for evaluating the "goodness" of any identified model is simulation and comparison with an input not used in the identification. For the evaluation, a 2.1-rad/sec sinusoidal pitch-axis input was used to drive both the baseline model and the identified model. The simulated time-historles were then compared.

Figure 6 shows the results of the simulation and comparison of the models. As done previously for the mathematically reduced models, only the primary axis (pitch rate) and cross-axis response (roll rate) are shown. Again, there 18 iery little difference in the primary-axis response, with major discrepancies occurring in the cross-axis response. The identified model using the conventional model structure is unable to predict the correct cross-axis response, being grossly in error. It does not even come close to the incorrect reduced model response of Fig. 3. The amended model, on the other hand, does show considerably better agreement with the actual baseline model response and, in fact, does not display the shortcomings of the mathematically reduced amended model (Fig. 3), which had the incorrect amplitude behavior.

Identified Derivatives and Eigenvalues. In addition to the direct comparison of the time-history responses, it was also desirable tn evaluate the stability derivatives and eigenvalues of the identified models. A selection of several important stability derivatives is shown in Fig. 7, where the identified derivatives are compared with the time-varying baseline model-perturbation derivatives.

The baseline reference derivatives are plotted in their 6-DOF time-varying perturbation form, where the initial value $(t=0)$ is identical to the coefficient of the $F_{B}$ submatrix of the 9-DOF baselfne model, and the steady-state value is the same as the mathematically reduced conventional 6-DOF quasi-stictic derivative. The 8-DOF identiffed derivative is also plotted as an equivalent 6-nnf time-varying perturbation derivative. Its initial value corresponds to thic sppropriate cnefficient of the identified $F_{B}$ matrix for the 8-DOF model, and the steady-stace value is that derivative value that would result should the 8-DOF identified model be mathematically reduced into quasi-static derivatives. The: conventional 6-Lúf stability derivatives are time-invariant and are represented by a constant-valued line across the plot.

The plots in Fig. 7 show that identification of parameters, using the conventional 6-DOF model structure, will yield derivative values that are quantitatively different from the conventional (i.e., mathematically reduced) quasistatic derivative (this was first noted in Ref. [6]). The identified derivatives are always smaller in magnitude than the conventional value, but are in all cases within the extremes of the fluctuations of the time varying baseline derivative. This indicates that for the data used in the identification, the rotor is not operating in its steady-state equilibrlum; rather, because of excitation from the control inputs and body motions, it is operating in a transient manner.

The 8-DOF time-varying derivatives generaliy attempt to duplicate the dynamic nature of the time-varying baseline derivative. In most cases, its 
steady-state values are closer to the conventional derivative (1.e., matherically reduced) values than the identified values from the conventional 6-DOF stability derivative model.

The eigenvalues for each of the identified models are shown in Fig. 8 (body roots only). All models compare favorably with the lower frequency baseline characteristic roots; however, there are marked discrepancies in the shortperiod roots. The short-period eigenvalues for the identif ied 8-DOF model show fairly good agreement with the baseline model roots, correctly estimating the natural frequency but slightly underestimating the damping. The elgenvalues of the model identified using the amended-model structure, although still perlodic, greatly overpredict the short-period damping, and are not in as good agreement with the baseline roots as they were for the amended reduced model in Fig. 5 . The eigenvalues of the model identif ied using the conventional model structure are aperiodic and provide even worse correlation.

It is interesting to note that the discrepancies in the short-period eigenvalues can be shown to be related to the low values of the identified derivatives. Since the trace of any square matrix (i.e., the sum of the diagonal elements) is equal to the sum of the eigenvalues of that matrix (see Ref. [14]), the sum of the damping derivatives $X_{u}, Y_{v}, Z_{w}, L_{p}, M_{q}$, and $N_{r}$ for a given 6-DOF model will be equal to the sum of the real parts of the eigenvalues of that model (the imaginary parts cancel out). Evidence of the lcwer parameter estimates can be seen in Fig. 8, where the mean of the real parts of the short-period eigenvalues (for either of the 6-DOF identified models) is $v$ isibly less than that for the baseline model. This is an indication that the accuracy of ilentification of the helicopter short-term response is 1ikely to have a strong effect on the values of the parameters identified.

\section{Conclusions}

The validity of several helicopter models that can be used for flightdynamics analysis has been investigated. The purpose of the study was to assess the effect of the rotor-flapping dynamics on the helicopter handling-qualities motion spectrum, and to evaluate various simplifled model representations. The effects of nonlinearities and additional degrees of freedom above the rotor $t i p-$ path-plane flapping dynamics were not included in the analysis.

Three models were investigated - the conventional 6-DOF model, which is composed of the conventional quasi-static stability derivatives; an amended 6-DOF model, which includes body-acceleration and control-rate derivatives; and the 8-DOF tip-path-plane tilt model, which is evaluated because it represents the next higher level of complexity above the 6-DOF stability-derivative models. Another model, a linear 9-DOF (fourteenth order) body + rotur model, which includes rotor advancing, coning, and regressing flapping modes, in addition to the fuselage/body modes of motion, was used as a baseline model. It was from this baseline model that the mathematically reduced models were generated, and it was against this model that all the lower-order models, Including the identif ied models, were evaluated.

System identification of models using each of the thiee model structures was undertaken in order to assess the effects of the higher-order rotor dynamics on parameter identification and to determine how well the parameters could be identified under these idealized circumstances. The identified derivatives provide an upper bound on the quality of results that can be obtained from flight-test stability derivative extraction using dynamic testing techniques. 
The following conclusions can be made about the mathematically I duced models:

1. The conventional quasi-static stability derivative model was found to have problems in predicting the short-perfod cross-axis response.

2. The amended model, which included the body-acceleration and controlrate derivatives, essentially doubled the usable bandwidth and resulted in better predictions of the helicopter cross-coupling dynamics.

3. The 8-DOF codel provirnd the best correlation with the baseline model dynamics.

The following conclusions can be made about the identifled models:

1. Parameters identified using the conventional model structure were grossly in error, and were unable to predict the helicopter cross-axis response.

2. Parameters identified using the amended model structure [eq. (13)] were able to predict accurately the-helicopter cross-coupling behavior.

3. The 8-DOF identified mode] provided little noticeable improvement over the amended model in the time-history comparisons, but did provide better agreement with the baseline short-period eigenvalues and with manv stability derivatives.

4. Derivatives identified under the conditions of this study were $a$. ntitatively different from the conventional derivatives currently used by helicopter flight dynamicists. True helicopter 6-DOF perturbation derivatives are time-varying, and identified derivatives reflect this by effectives "sveraging" the time variations of the derivative.

\section{REFERENCES}

1. G. H. Bryan, Stability in Aviation, M.cMillan and Co., London, 1911.

2. K. H. Hohenemser, Dynamic stability of a helicopter with hinged rotor blades, NACA TM-907, Sept. 1939.

3. C. W. Ellis, Effects of rotor dynamics on helicopter automatic control system requirements, Aeronautical Engineering Review, July 1953.

4. R. L. Heimbold and D. C. Griffith, Symthesis of an electromechanical control system for a compound hingeless rotor helicopter, Journal of the American Helicopter Society, Apr. 1972.

5. W. E. Hall and A. E. Bryson, The inclusion of rotor dynamics in controller design for helicopters, AIAA Paper 72-778, 4 th Aircraft Design, Flight Test and Operations Meeting, Aug. 1972.

6. J. A. Molusis, Analytical study to define a helicopter stability derivative extraction method, NASA CR-132371, Vol. 1, May 1973.

7. P. G. Hamel, Determination of aircraft dynamic stability and control parameters from flight testing, Dynamic Stability Parameters, AGARD-LS-114, Mar. 1981. 
8. D. G. Gould and W. G. Hindson, Estinates of the lateral-directional stability derivatives of a helicopter from flight measurements, National Research Council of Canadá Aeroneutical Report LR-572, Dec. 1979.

9. K. H. Hohenemser, Hingeless rotorcraft flight dynamics, AGARD-AG-197, Sept. 1974.

10. J. A. Molusis, Rotorcraft derivative identification from analytical mod.' is and flight test data, Methods for Aircraft State and Parameter Identification, AGARD-CP-172, Nov. 1974.

11. K. H. Hohenemser and S. K. Yin, On the use of first order rotor dynamics in multiblade coordinates, Preprint 831, American Helicopter Society 30th Annual National Forum, May 1974.

12. S. J. Briczinski and D. E. Cooper, Flight investigation of rotor/ vehi, Le state feedback, NASA CR-132546, Jan. 1975.

13. J. Kaletka and 0. Rix, Aspects of system identification of helicopters, Paper 15, Third European Rotorcraft a.dd Powered Lift Forum, Sept. 1977.

14. P. M. DeRusso, R. J. Roy, and C. M. Close, State Variables for Engineers, John Wiley and Sons, Inc., New York, 1965.

TABLE 1.- ROTOR + BODY MODELS

\begin{tabular}{|c|c|c|c|c|}
\hline $\begin{array}{l}\text { Ro:or }+ \text { body } \\
\text { model }\end{array}$ & Rotor model & Rotor states & Rotor modes & Order \\
\hline 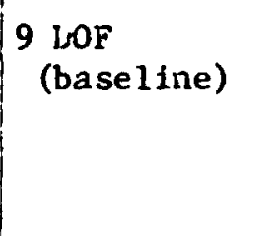 & $\begin{array}{l}\text { Sec } \cdot \text { ld-order } \\
\text { p-path-plane } \\
\text { dynamics }\end{array}$ & $\underline{x}_{R}=\left[\beta_{0}, \beta_{1}, B_{1}, \beta_{0}, \dot{B}_{1}, \dot{B}_{1}\right]^{T}$ & $\begin{array}{l}\text { Advancing } \\
\text { flapping } \\
\text { Coning } \\
\text { Regressing } \\
\text { flapping }\end{array}$ & $\begin{array}{c}14 \\
(8+6)\end{array}$ \\
\hline Coning & $\begin{array}{l}\text { cacond-order } \\
\text { coning } \\
\text { First-ordar } \\
\text { ti path-plane } \\
\text { tilt }\end{array}$ & $\underline{x}_{R}=\left[\beta_{0}, \beta_{1}, \beta_{1 s}, \dot{\beta}_{0}\right]^{T}$ & $\begin{array}{l}\text { Coning } \\
\text { Regressing } \\
\text { flapping }\end{array}$ & $\begin{array}{c}12 \\
(8+4)\end{array}$ \\
\hline 8 DOF & $\begin{array}{l}\text { Tip-path-plane } \\
\text { tilt only } \\
\text { (first order } \\
\text { each DOF) }\end{array}$ & $\underline{x}_{R}=\left[B_{1_{c}}, B_{1_{s}}\right]^{T}$ & $\begin{array}{l}\text { Regressing } \\
\text { flapping }\end{array}$ & $\begin{array}{c}10 \\
(8+2)\end{array}$ \\
\hline
\end{tabular}


ORICWNAL PACE is

OF POOR QUALIT
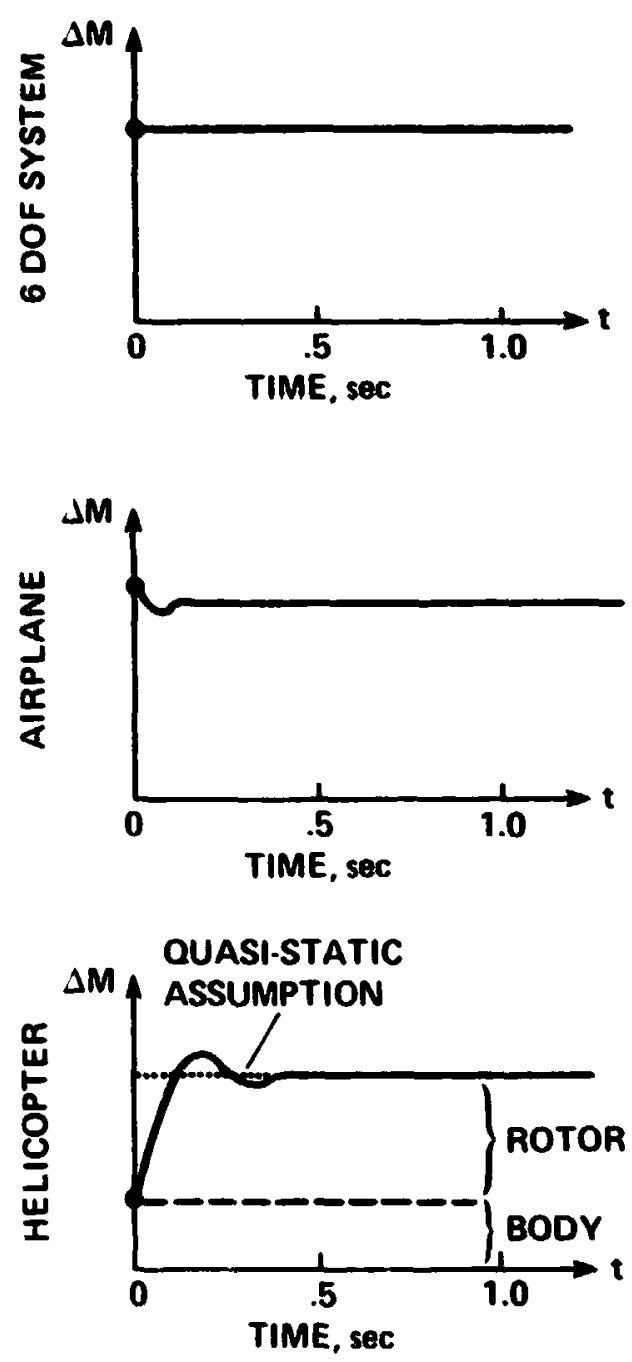

Fig. 1. $\Delta M$ response to a constant-perturbation $\Delta w$ for an 1 deal six-degreeof-freedom system, an airplane, and a helicopter. 


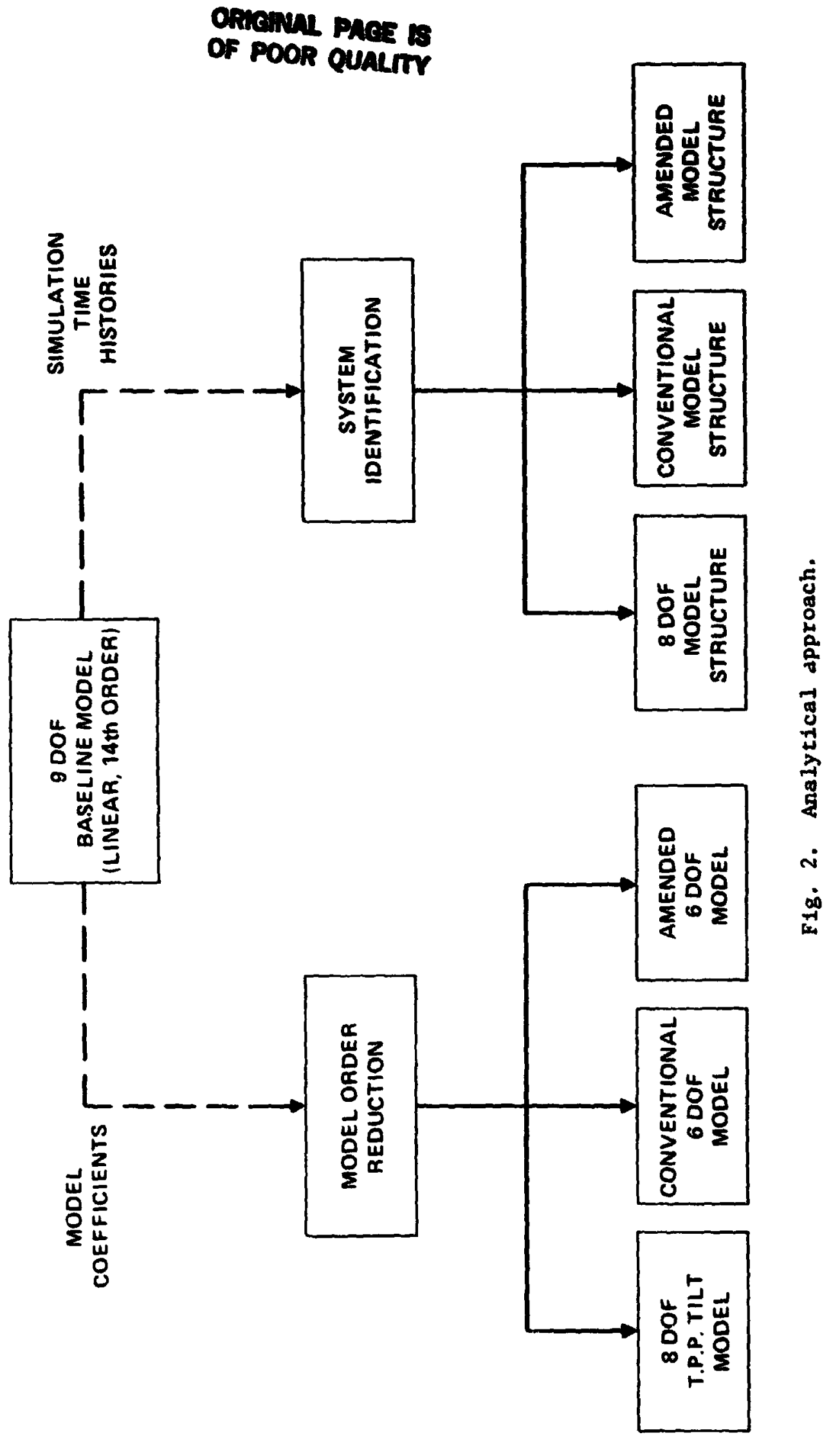




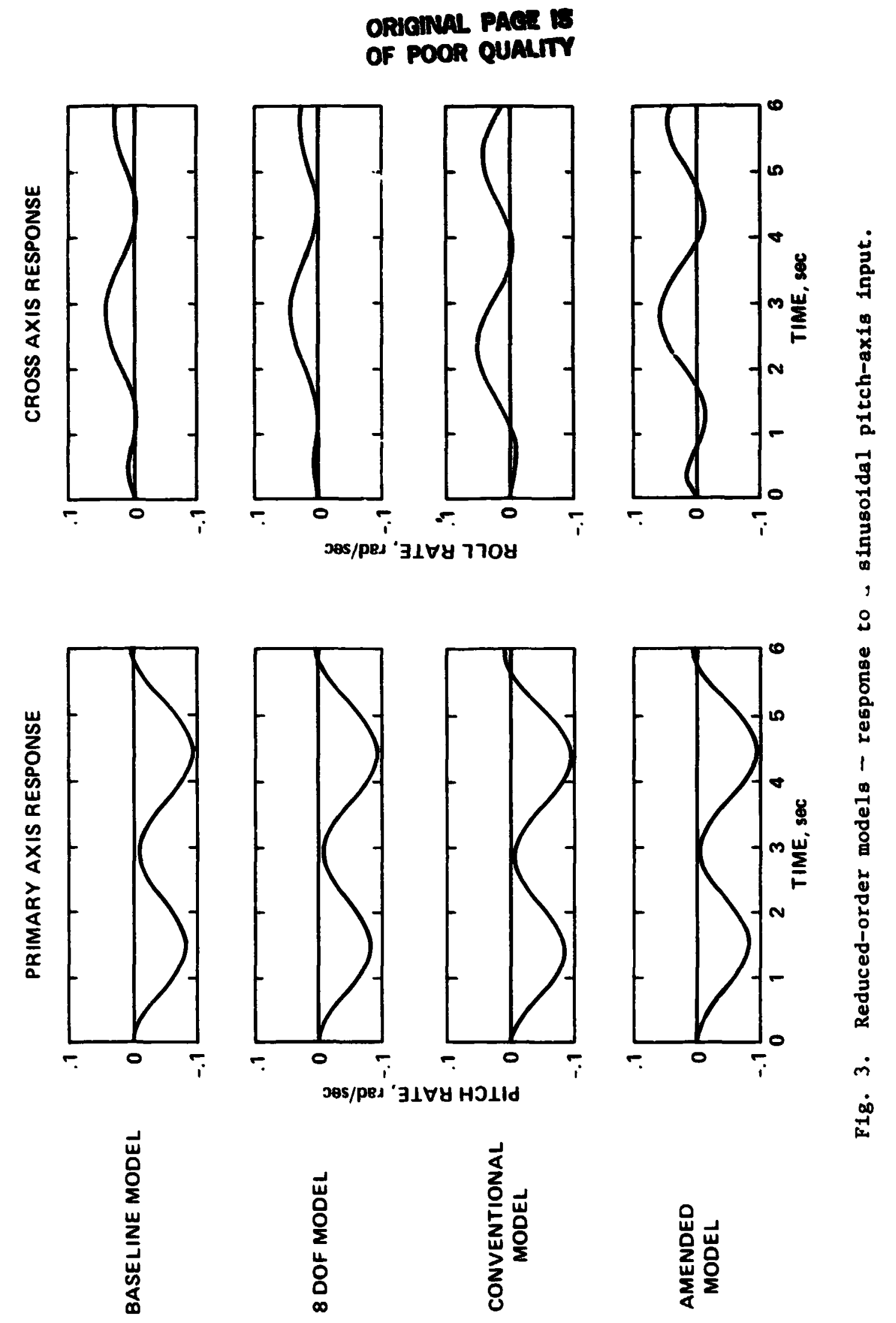


ORIGINAL PAGE IS

OF POOR QUALITY
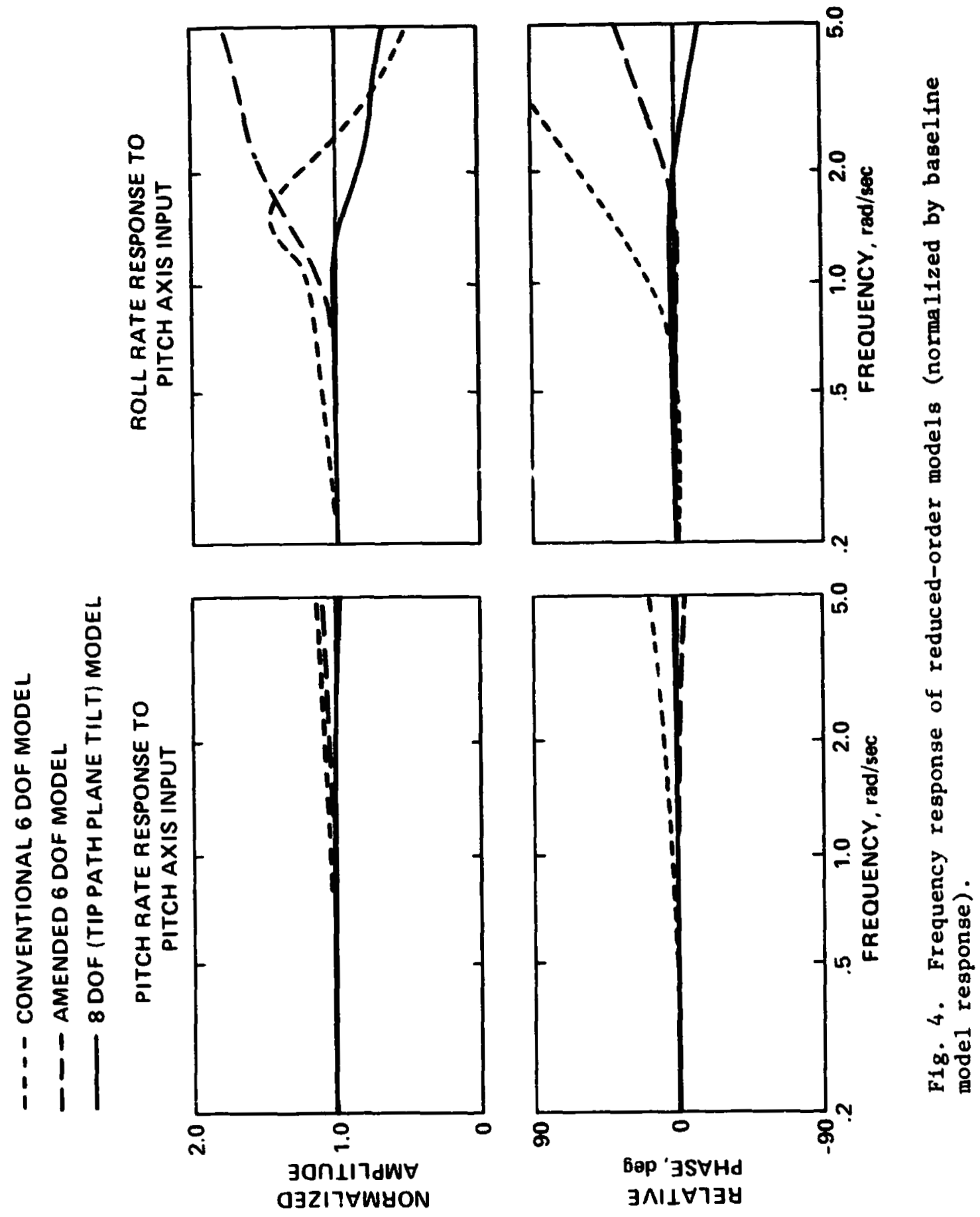
ORIGINAL PAGE IS

OF POOR QUALITY

$\times$ BASELINE MODEL*

$\triangle 8$ DOF MODEL*

๑ CONVENTIONAL MODEL

$\odot$ AMENDED MODEL

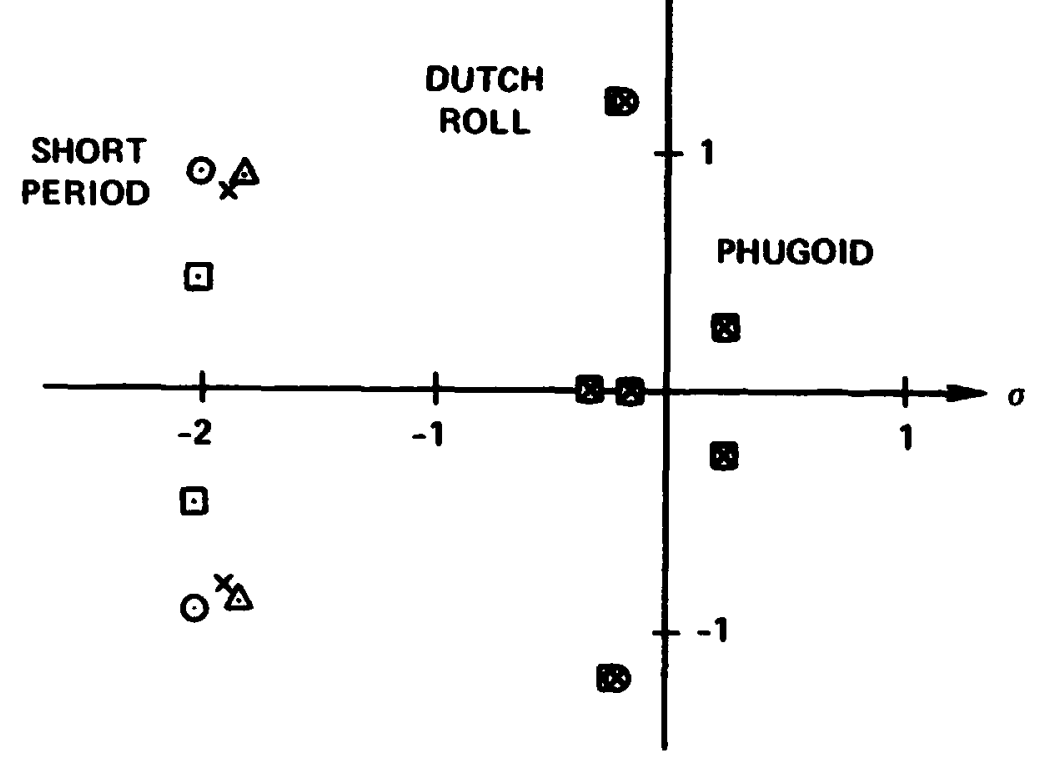

- FUSELAGE/BODY ROOTS ONLY ROTOR FLAPPING MODES NOT SHOWN

Fig. 5. Eigenvalues of mathematically reduced models. 
ORIGINAL PAGE IS

OF POOR QUALTY
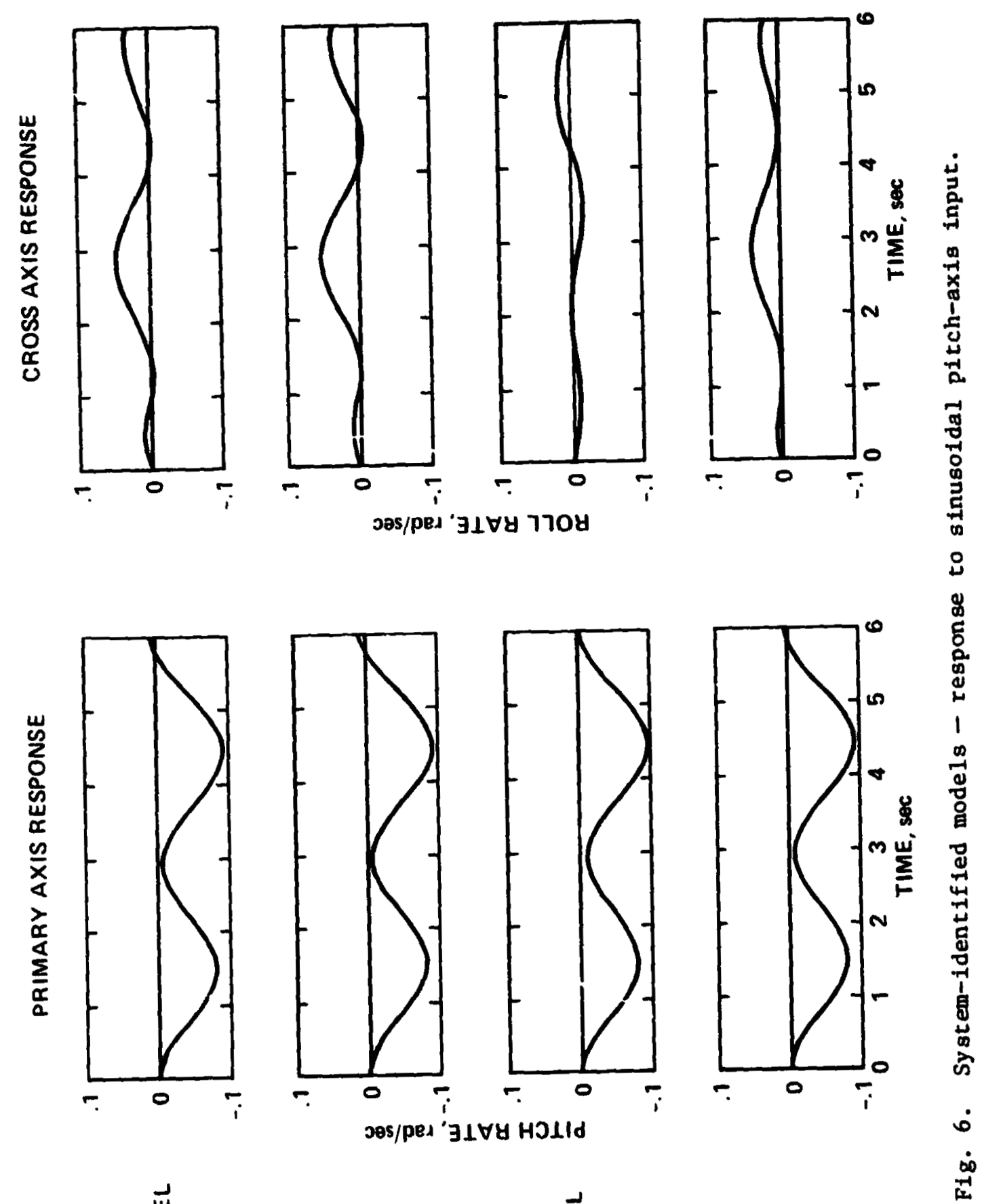

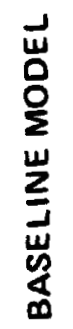

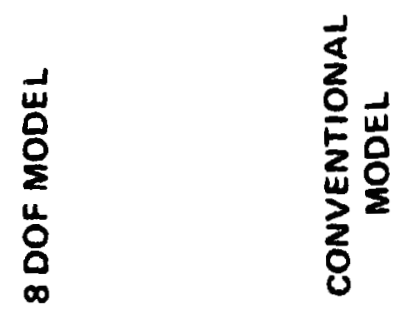

㞺耑 
ORIGINAL PAGE IS

OF POOR QUALITY

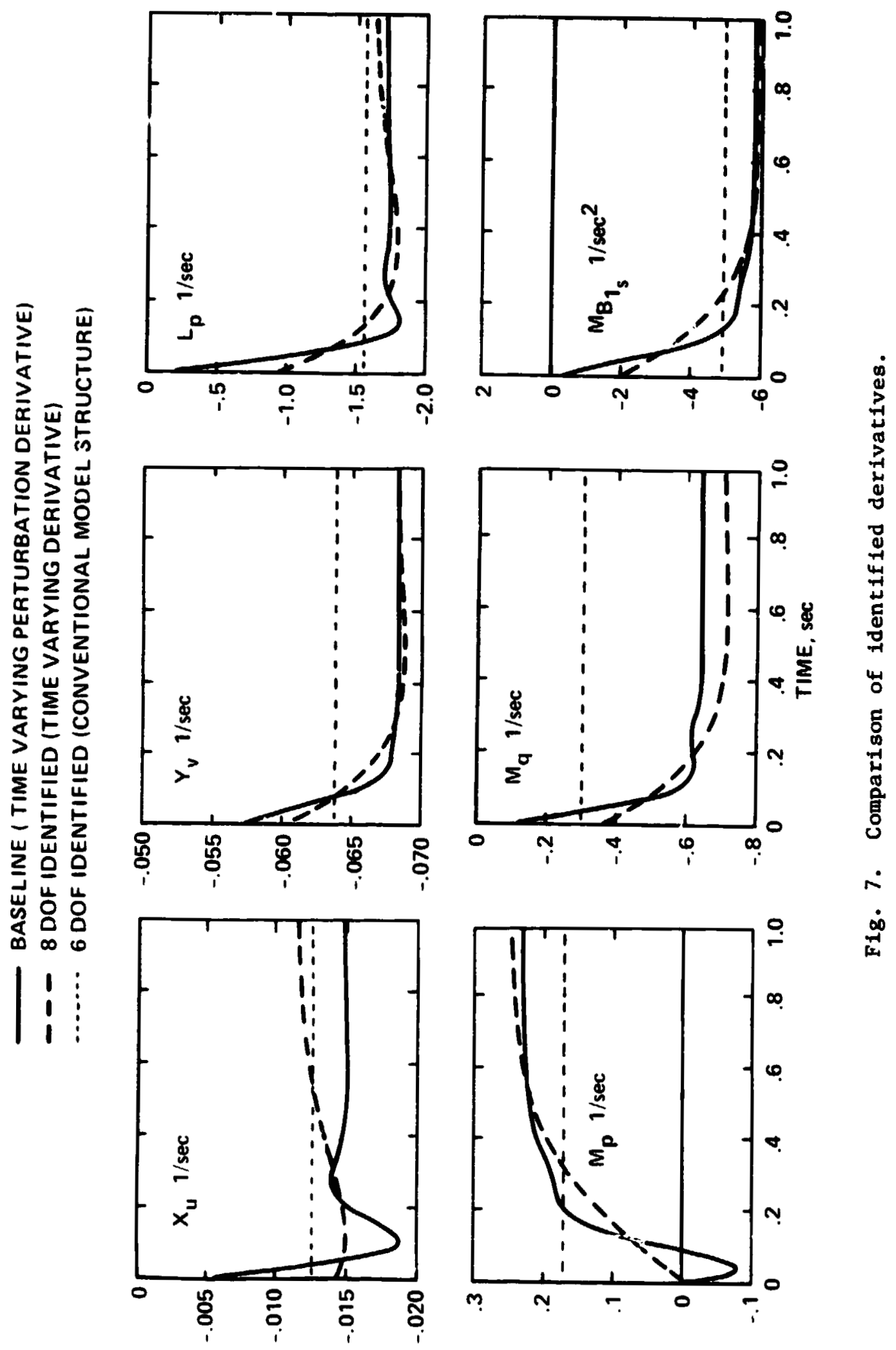


ORIGINAL PAGE IS

OF POOR QUALITY

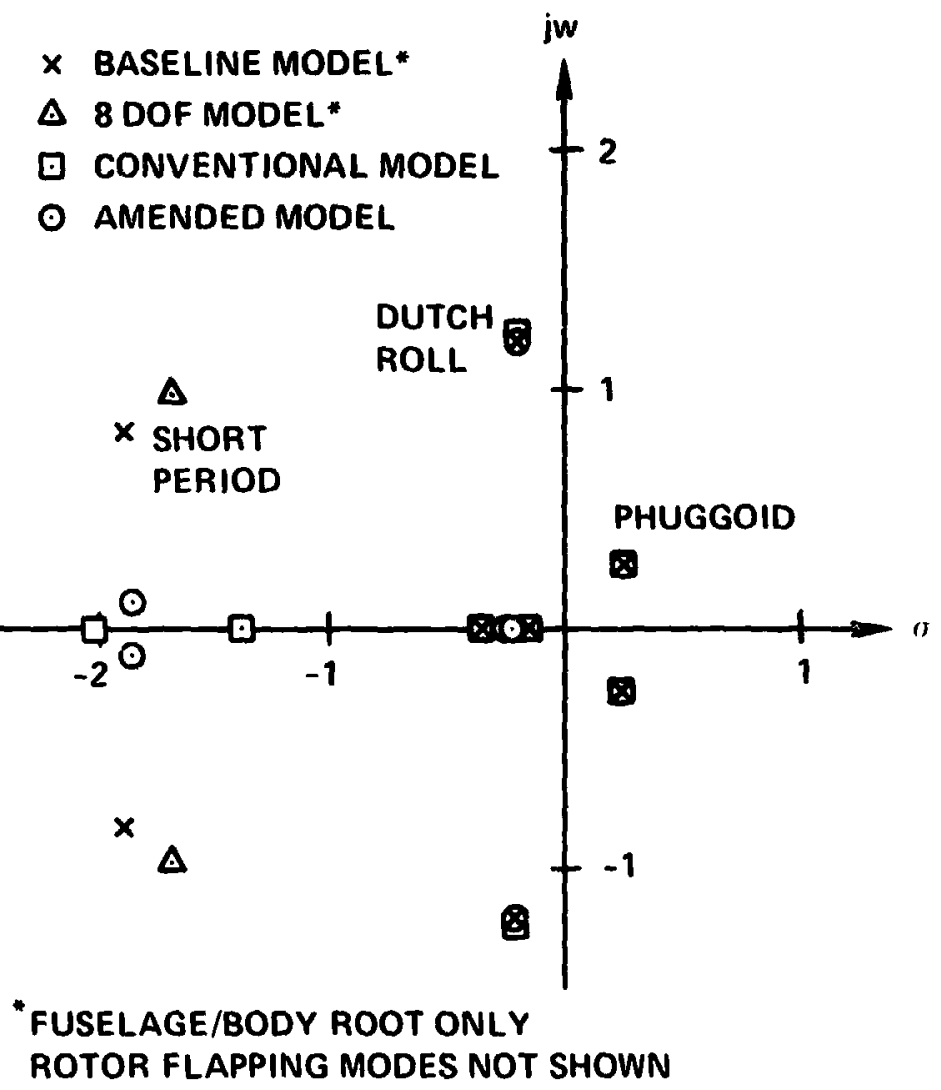

Fig. 8. Eigenvalues of identified models. 